\title{
The Solubility of Oxygen in $\delta$-Iron*
}

\author{
By Akihiko KUSANO, ${ }^{* *}$ Kōin ITŌ, ${ }^{* * *}$ and Kōkichi SANO ${ }^{* * *}$
}

\begin{abstract}
Synopsis
The authors measured the distribution equilibria of oxygen between liquid iron and solid by directly equilibriating the liquid and solid phases.

First, the melting point of purified iron $\left(1538^{\circ} \mathrm{C}\right)$ and the monotectic point $\left(1528^{\circ} \mathrm{C}, 0.16^{\circ} \mathrm{O}\right)$ were confirmed by analyzing the cooling curve. Next, the liquidus line was confirmed and then the solidus line was determined. Both lines were nearly linear. Finally, the partition ratio of oxygen between liquid iron and solid was determined to be 0.076; it was almost independent on temperature. Thus, the solubility of oxygen in $\delta$ iron was determined to be $0.012 \%$ at $1528^{\circ} \mathrm{C}$, and the heat of fusion of iron was found to be $3450 \mathrm{cal} / \mathrm{mole}$, which was in good agreement with the presently known value.
\end{abstract}

\section{Introduction}

Oxygen is one of the most important impurities present in iron or steel, mainly forming oxide inclusions. Therefore, it is often desirable to know the solubility of oxygen in solid iron with a reasonable accuracy, but past investigations have brought forth highly discordent results. The solubility of oxygen in $\alpha$-iron was studied by Seybolt, ${ }^{1)}$ Sifferlen, ${ }^{2)}$ and others, and that in $\gamma$-iron was done by Kitchener et al. ${ }^{3}$ ) Those were more recently studied by Ihida et al., ${ }^{4}$ ) Swisher and Turkdogan, ${ }^{5)}$ and others. Tankins and Gokcen, ${ }^{6)}$ and Hepworth et al. ${ }^{7)}$ held the zone-refined iron in $\mathrm{H}_{2}-\mathrm{H}_{2} \mathrm{O}$ gas mixture at constant temperatures for many hours and determined the solubility of oxygen in $\delta$-iron from the equilibrium of gas reaction. The solubility of oxygen at $1528^{\circ} \mathrm{C}$ was found to be $0.0088 \%$ by Hepworth et al. ; it was estimated to be about $0.03 \%$ from the results by Tankins and Gokcen. Results are discrepant not only in $\delta$-iron but also in $\alpha$-iron and $\gamma$-iron. As shown in many literatures, this is attributed to the presence of oxidizable impurities in solid iron, such as silicon, manganese, aluminum, etc.

In the present work, the authors carried out the experiments by lowering the crucible little by little in a Tamman furnace along its temperature gradient and letting the molten iron solidify unidirectionally; the equilibrium between solid iron and liquid was thus assured. From the partition ratio of oxygen, the solubility of oxygen in $\delta$-iron was determined.

\section{Experimental Apparatus}

A schematic diagram of the gas purifier used throughout the experiments is shown in Fig. 1. Argon gas was passed through a mercury-overflow bulb and a flowmeter; it was then freed from the moisture with silica gel, natron asbestos, and phosphorus pentoxide.

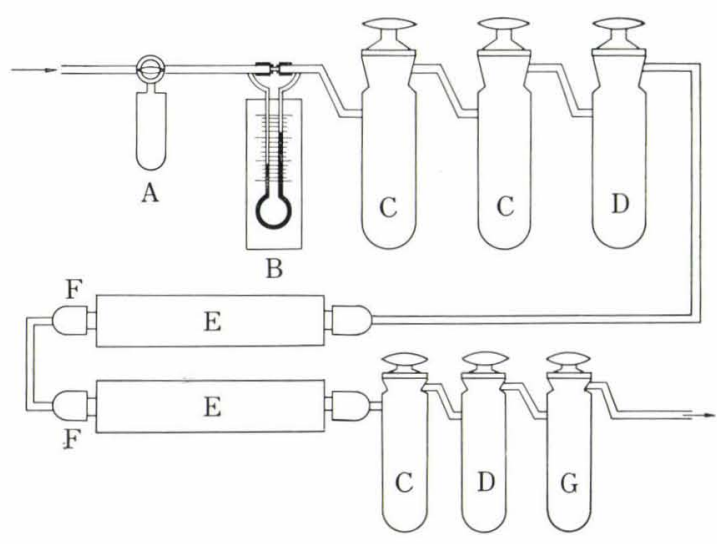
(A) Hg-overflow bulb
(E) Mg-chip furnace
(B) Gas flowmeter
(C) Silica gel
(F) Water cooling jacket
(D) Natronasbestos
(G) $\mathrm{P}_{2} \mathrm{O}_{5}$

Fig. 1. Apparatus used for Ar-gas purification

Argon was further passed through magnesium chip furnaces to remove oxygen. The refined argon was then led into a Tamman furnace. Schematic cross section of the Tamman furnace used for the melting experiments is shown in Fig. 2. Through the cap of furnace, two semi-fused alumina tubes $(6 \phi \times 4 \phi \times$ $600 \mathrm{~mm}$ ) with one end closed, were inserted into the furnace. One of them, which sheathed a $5 \mathrm{RhPt}$ $20 \mathrm{RhPt}$ thermocouple, was used to probe the position of solid-liquid interface and at the same time to measure the equilibrating temperature, while the other sheathing a $13 \mathrm{RhPt} / \mathrm{Pt}$ thermocouple was connected to a temperature controller. Fused magnesia crucible $(36 \phi \times 26 \phi \times 100 \mathrm{~mm})$ was used for most of the experimental runs, but for those of low oxygen concentrations, semi-fused alumina crucible $(26 \phi \times 22 \phi \times$ $90 \mathrm{~mm}$ ) was used. The crucible was manually moved by a lift attached to the bottom of furnace.

The temperature distribution of the furnace was measured beforehand and the position and extent of the uniform temperature zone was known. The temperature of the furnace was controlled by a controller in conjunction with the thermocouple. It was confirmed that the temperature fluctuation was less than $\pm 2{ }^{\circ} \mathrm{C}$ during the experiments at about $1550^{\circ} \mathrm{C}$.

\section{Experimental Procedure}

The electrolytic iron used for this investigation

* Originally published in Tetsu-to-Hagané, 54 (1968), 553 in Japanese. English version received July $31,1969$.

** Department of Metallurgy, Faculty of Engineering, Nagoya University. Now at Tobata Works, Yawata Iron \& Steel Co., Ltd., Kitakyushu 805.

*** Department of Metallurgy, Faculty of Engineering, Nagoya University, Chikusa-ku, Nagoya 464. 


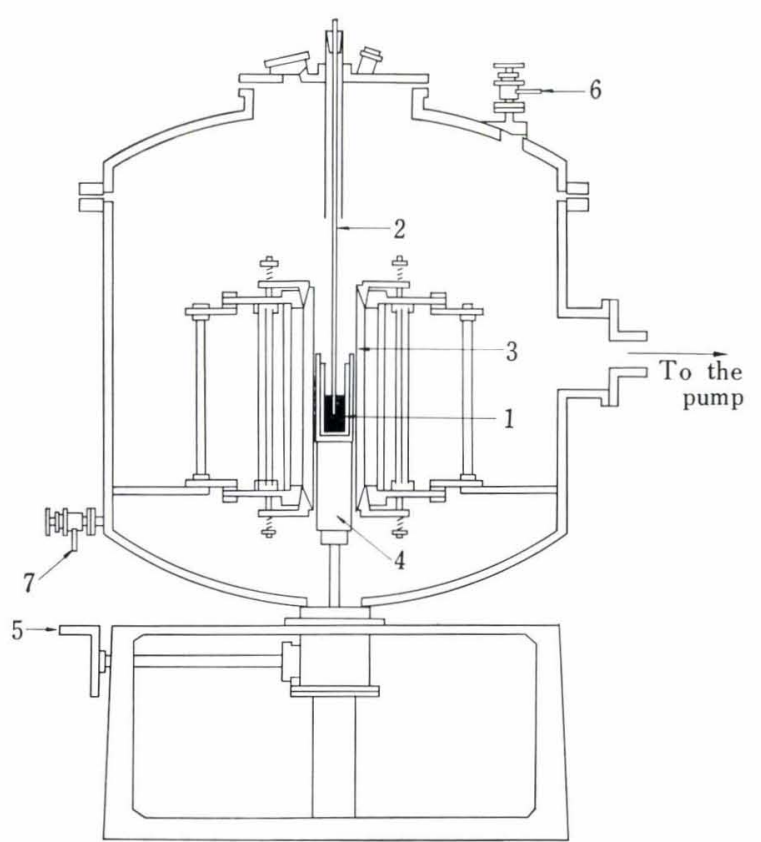

(1) $\mathrm{MgO}$ crucible

(5) Handle

(2) Thermocouple

(6) Gas inlet valve

(3) Heater

(4) Crucible supporter

Fig. 2. Tamman furnace used for experiment

was generally of the composition of:

$$
\begin{aligned}
& \mathrm{Fe}>99.95 \%, \mathrm{C}<0.005 \%, \mathrm{~S}<0.005 \%, \mathrm{P}<0.003 \%, \\
& \mathrm{Mn}<0.006 \%, \mathrm{Si}<0.005 \%, \mathrm{Cu}<0.004 \% .
\end{aligned}
$$

A 200 to $230 \mathrm{~g}$ of this iron (when the iron was melted the depth was about 55 to $60 \mathrm{~mm}$ ) and a suitable amount of ferric oxide (99\%) were charged in a crucible, which was placed in the uniform temperature zone of the furnace, and argon gas was introduced into the furnace after the furnace was once evacuated. The pressure of argon in the furnace was kept about $5 \mathrm{~mm} \mathrm{Aq} \mathrm{higher} \mathrm{than} \mathrm{the} \mathrm{atmospheric} \mathrm{pressure,} \mathrm{and}$ heating was started keeping the flow of argon on molten iron constant. After the melt down, the molten iron was held at about $1700^{\circ} \mathrm{C}$ for $40 \mathrm{~min}$ to dissolve the oxide perfectly.

Then the temperature of the melt was lowered to about $1570^{\circ} \mathrm{C}$; it was lowered slowly further while measuring the temperature. When the proper temperature $\left(1540^{\circ}\right.$ to $1550^{\circ} \mathrm{C}$ ) was reached, the crucible was lowered $10 \mathrm{~mm}$ so that the solid phase might grow at the bottom of crucible; this was checked by the probe and temperature measurement. If the solid had not grown, the crucible was raised back, and the temperature was lowered further, until the solid phase grew without failure when the crucible was lowered $10 \mathrm{~mm}$.

After about $15 \mathrm{~min}$, the position of solid surface was probed by the sheathed thermocouple, and crucible was lowered further $5 \mathrm{~mm}$. After another $15 \mathrm{~min}$, the solid surface was again probed to make it certain that it did not move. Then two pairs of thermocouple were set at $8 \mathrm{~mm}$ above the surface : one of them was connected to a temperature controller and the incidental temperature was maintained, while the other is used to measure the temperature during the experiment. After the controller was set, a sample for oxygen analysis was taken from the melt by means of a fused silica tube (3 $\mathrm{mm}$ i.d.).

Then, the crucible was lowered some distance and kept there for a predetermined period of time. This operation was repeated several times until a solid sample of about $8 \mathrm{~mm}$ thickness was formed under liquid-solid equilibrium. Then the solid surface was probed, and a sample for oxygen analysis was again taken from the residual melt. Finally, some 1 to $2 \mathrm{~g}$ of $\mathrm{FeS}^{*}$ was dropped into the melt through a silica tube $(10 \mathrm{~mm}$ i.d.), the crucible was lowered immediately to the bottom of the furnace, the current to the furnace turned off, and the specimen was quickly solidified there.

The last solid-liquid interface was observable with naked eye in the lengthwise cross section of the specimen ingot, nevertheless sulfur print was made for confirmation as shown in Photo. 1. A photomicrograph of the vicinity of the interface is shown in Photo. 2, where it is possible to distinguish the previous solid and liquid phases at the end of experiment.

The sample for oxygen analysis of the solid phase was machined out of the middle part of the $8 \mathrm{~mm}$ sample to $5 \mathrm{~mm}$ length. The concentration of oxygen was determined by the vacuum fusion method.

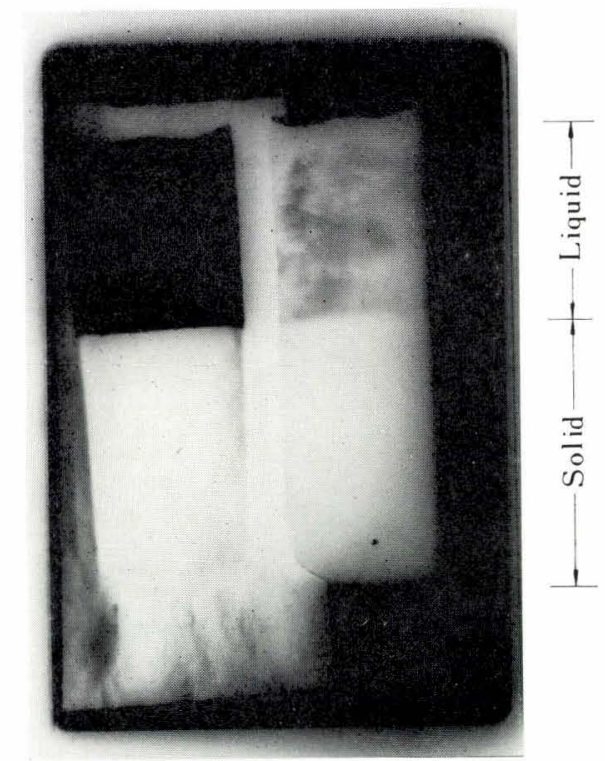

Photo. 1. Macrophotograph of sulfur print (left) and its specimen (etched by $1 \% \mathrm{H}_{2} \mathrm{SO}_{4}$, right)

\section{Experimental Results}

\section{On the Liquidus Line}

Although the relation between the temperature and solubility of oxygen in liquid iron has been studied in

* The FeS used in this experiment was made from powdery iron and sulfur by heating the mixture at about $700^{\circ} \mathrm{C}$ in $\mathrm{H}_{2}-\mathrm{Ar}$ ( 1 to 30 ) for $1 \mathrm{hr}$; it was about $20 \% \mathrm{~S}$. 


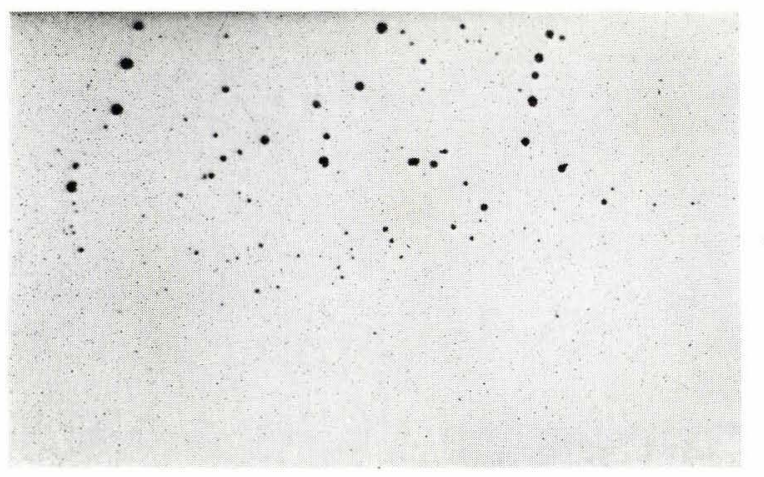

Photo. 2. Microphotograph of the liquid-solid interface $(\times 100)(2 / 3)$

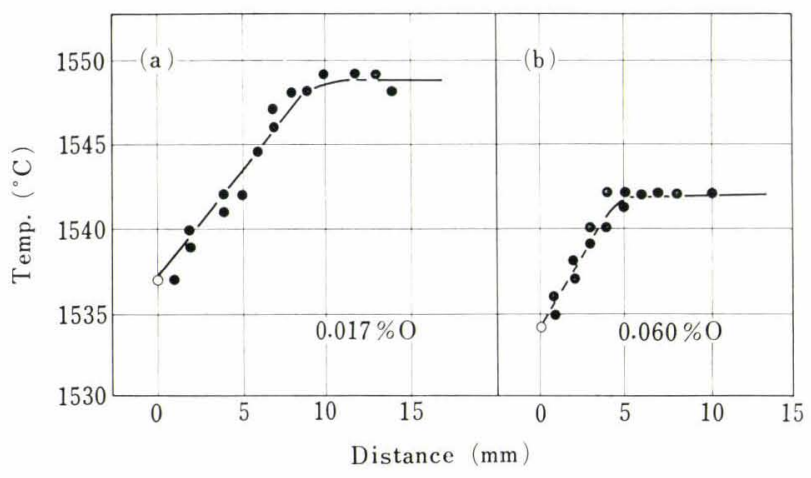

Fig. 3. The temperature distribution in liquid iron; open circles indicate the extrapolated value.

detail, the liquidus line in the region below $0.16 \% \mathrm{O}$ has remained unestablished. Therefore, the liquidus line was determined anew as follows.

After the solid-liquid equilibrium was attained by the same procedure as mentioned above, the thermocouple was raised away from the solid-liquid interface by $1 \mathrm{~mm}$ each time so as to indicate the temperature distribution in the liquid. Two typical results are shown in Fig. 3. It is clearly seen that the temperature increases linearly with increasing distance from the interface until it becomes nearly constant. Extrapolation of the line linearly back to the zero distance, i.e., to the position of the interface, gives a temperature on the ordinate, which should be the temperature where the solid and liquid are equilibrated. A sample taken from liquid iron near the interface in a fused silica tube gives, on the other hand, the oxygen concentration in liquid iron under the equilibrium.

Further, it was verified that the oxygen concentration in liquid iron varied very little. In one experiment, for example, it was $0.0615 \%, 0.0625 \%$, and $0.0614 \%$ at $2 \mathrm{~mm}, 25 \mathrm{~mm}$, and $40 \mathrm{~mm}$, respectively, above the interface. As the temperature difference was less than $10^{\circ} \mathrm{G}$ in the liquid, the concentration of oxygen in melt should be regarded to be constant within the experimental error.

Finally, the peritectic temperature was determined with irons containing $0.022 \% \mathrm{O}$ and $0.14 \% \mathrm{O}$ by the arrest in their cooling curve : it was found to be $1528^{\circ}$ $\pm 1^{\circ} \mathrm{C}$.

The relation between the oxygen concentration in

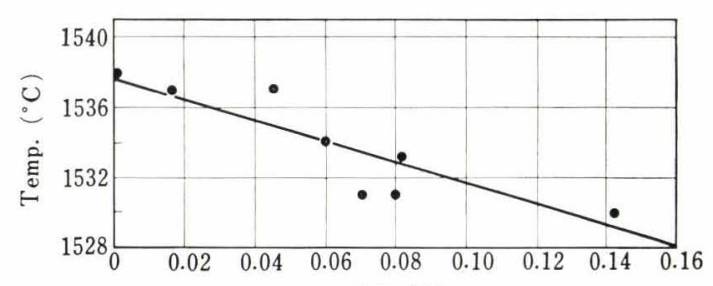

[0] $(\%)$

Fig. 4. The liquidus line as determined by the extrapolation of the temperature distribution in liquid phase

melt and the temperature of solid-liquid interface thus determined is shown in Fig. 4, where the straight line was fitted by the least square method. In the present work, the melting point of pure iron was found to be about $1538^{\circ} \mathrm{C}$.

\section{Solubility of Oxygen in $\delta$-Iron}

In the first series of experiments using molten iron of 0.08 to $0.14 \%$, it was found that the oxygen partition ratio was influenced by the unit distance by which the crucible was lowered at a time and the period of time that the iron was held at that position. Some of the results are shown in Fig. 5. $L_{0}$, the partition ratio being given by

$$
L_{\mathrm{o}}=C_{s} / C_{l}
$$

where, $C_{s}$ : the oxygen concentration in solid iron, and

$C_{l}$ : the mean concentration of oxygen in liquid iron at the start and at the end of the run.

It will be seen in Fig. 5 that $L_{\mathrm{o}}$ approaches to 0.76 , the equilibrium value, as the period of time of holding increases: when the unit distance is $1 \mathrm{~mm}$, some $10 \mathrm{~min}$ is sufficient to obtain the equilibrium, but for $2 \mathrm{~mm}$ at least $20 \mathrm{~min}$ is necessary. However, when the unit distance was greater than $4 \mathrm{~mm}$, the oxygen partition ratio did not reach the equilibrium value, even if the holding time was very long. Consequently, 1 to $2 \mathrm{~mm}$ was judged to be favorable for the unit distance.

The above-mentioned results were obtained in a range of relatively high oxygen concentration $(0.08$ to $0.14 \% \underline{\text { O }}$ ). Next, experiments were also done in a range of low oxygen concentration in order to study the

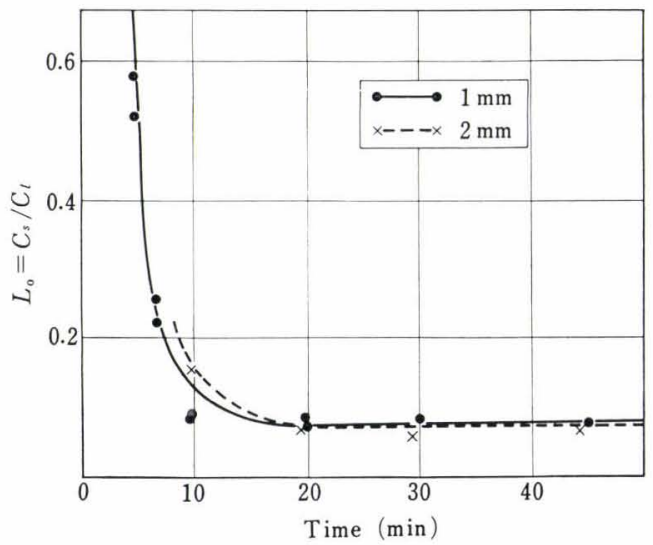

Fig. 5. The effect of the holding time on the partition ratio of oxygen 
effect of oxygen concentration on the partition ratio. The results are shown in Fig. 6, where the concentration of oxygen in solid phase are plotted against that in liquid phase arranged on the liquidus line given in Fig. 4.

As shown in Fig. 6, the partition ratio is independent of the concentration of oxygen in liquid phase, and the solidus line is linear. As the concentration of oxygen in solid phase at $1528^{\circ} \mathrm{C}$, the peritectic temperature, is $0.012 \%$, and that in liquid phase $0.16 \%$ the partition ratio is then $0.012 / 0.16=0.075$. This is in an excellent agreement with the result in Fig. 5, which gives $L_{0}=0.076$. Therefore, it is concluded here that the solubility of oxygen in $\delta$-iron is $0.012 \%$ at $1528^{\circ} \mathrm{C}$ and that the partition ratio of oxygen is 0.076 .

\section{Discussion}

In Table 1, the present result of the solubility of oxygen in $\delta$-iron at $1528^{\circ} \mathrm{C}$ is compared with those determined by other investigators. ${ }^{6,7)}$ As pointed out by Hepworth et al., ${ }^{7)}$ the value by Tankins and Gokcen $^{6)}$ involves some uncertainties as follows :

(1) the oxidizable impurities in their iron might have given rise to 20 to $30 \mathrm{ppm}$ excessive oxygen,

(2) the thin foil specimen they used would have readily gained or lost oxygen unless quenching was exceptionally fast, and

(3) the high specific surface area of the foil would have made the vacuum fusion analysis more erroneous.

The value given by Hepworth et al., ${ }^{7)}$ on the other hand, is lower than the present result. This discrepancy, though it is not very great, can be due to the difference in the purity between their zone-refined iron and present work's electrolytic iron. This, however, is unlikely because the contents of oxidizable impurities of this work's iron were so small that exact quantitative analysis was impossible as follows :

$\begin{array}{clc}\text { Impurity } & \text { Analytical method } & \text { Result } \\ \mathrm{Al} & \text { Aluminon absorptiometric } & \text { Trace } \\ \mathrm{Si} & \text { Molybdenum blue absorptiometric }\end{array}$ $<0.003 \%$

The real cause of this discrepancy should then be attributed to the experimental method they employed, i.e., the solid diffusion method, and the fewness of data point. In the former, it might have been that their rod specimens were not brought to equilibrium, and in the latter, their value is an extrapolation at $1528^{\circ} \mathrm{C}$ of only two measurements at $1450^{\circ}$ and at $1510^{\circ} \mathrm{C}$. Either would have yielded a result that is smaller than the true value.

The result of the present work may be examined by the value of the heat of fusion. Applying diagram determined in present work,

$$
L_{f}=R T^{2} d \ln a_{\mathrm{Fe}} / d T
$$

where, $L_{f}:$ the heat of fusion of purified iron (cal/mole),

$R:$ the gas constant,

$T$ : the absolute temperature, and

$a_{\mathrm{Fe}}:$ the activity of iron.

As the concentration of oxygen is very low,

$$
a_{\mathrm{Fe}}=\mathcal{N}_{\mathrm{Fe}}
$$

Substituting this into Eq. (2) and integrating, one gets ${ }^{8)}$

$$
\frac{L_{f}\left(T_{\mathrm{o}}-T\right)}{R T T_{\mathrm{O}}}=\ln \frac{\mathcal{N}_{\mathrm{Fe}}^{\prime}}{\mathcal{N}_{\mathrm{Fe}}}
$$

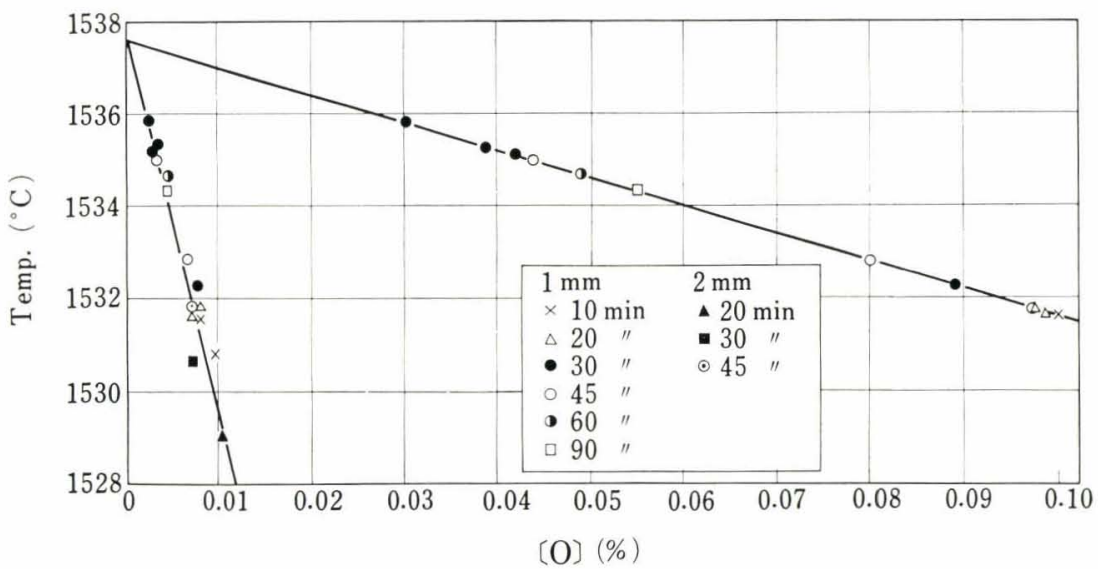

Fig. 6.

The relation between the concentrations

\begin{tabular}{|c|c|c|c|c|}
\hline \multirow{2}{*}{ Investigator } & \multicolumn{3}{|c|}{ Experimental method } & \multirow{2}{*}{$\begin{array}{c}\text { Solubility at } 1528^{\circ} \mathrm{C} \\
(\%)\end{array}$} \\
\hline & Specimen & Atmosphere & $\begin{array}{l}\text { Equilibration time } \\
\qquad(\mathrm{hr})\end{array}$ & \\
\hline $\begin{array}{l}\text { E.S. Tankins and } \\
\text { N.A. Gokcen }{ }^{6)}\end{array}$ & $\begin{array}{l}\text { Spiral foil of } \\
1 / 4 \mathrm{~mm} \text { thickness }\end{array}$ & $\mathrm{H}_{2}-\mathrm{H}_{2} \mathrm{O}$ & About 5 & 0.032 \\
\hline $\begin{array}{l}\text { M.T. Hepworth, } \\
\text { R.P. Smith, and } \\
\text { E.T. Turkdogan }{ }^{7)}\end{array}$ & $\begin{array}{l}\text { Rod of } \\
1 / 3 \text { inch dia., } \\
1.5 \text { inch long }\end{array}$ & $\mathrm{H}_{2}-\mathrm{H}_{2} \mathrm{O}-\mathrm{Ar}$ & 10 to 24 & 0.0088 \\
\hline Present work & \multicolumn{3}{|c|}{ Liquid-solid equilibrium } & 0.012 \\
\hline
\end{tabular}
of oxygen in solid and liquid phase

Table 1. Comparison between the present work and the other experimentations 
where, $T_{0}$ : melting point of pure iron,

$\mathcal{N}_{\mathrm{Fe}}^{\prime}$ : the molar fraction of iron in solid phase under equilibrium at $T$, and

$\mathcal{N}_{\mathrm{Fe}}: \quad$ the molar fraction of iron in liquid phase under equilibrium at $T$.

Thus the heat of fusion of pure iron $L_{f}$ can be calculated from the data shown in Fig. 6, which gives $L_{f}=3450 \mathrm{cal} / \mathrm{mole}$. This value is in good agreement with the presently known values $(3630 \pm 600 \mathrm{cal} /$ mole). ${ }^{9)}$

Finally, the phase diagrams of $\mathrm{Fe}-\mathrm{O}$ system due to Hepworth et al., ${ }^{7)}$ and Tankins and Gokcen ${ }^{18)}$ are compared with that of the present work in Fig. 7.

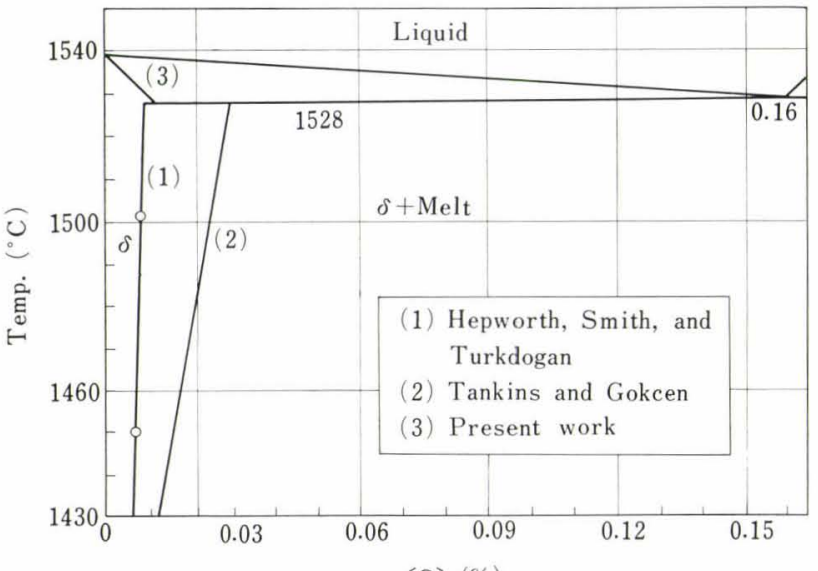

[O] $(\%)$

Fig. 7. The phase diagram of $\mathrm{Fe}-\mathrm{O}$ system. The present work is compared with the other investigations

\section{Conclusion}

By lowering a crubible little by little under purified argon gas in a Tamman furnace, the equilibrium be- tween solid iron and liquid with regard to oxygen was attained. It was found that:

(1) At the monotectic point in the $\mathrm{Fe}-\mathrm{O}$ system the concentration of oxygen is $0.16 \%$, and the temperature is $1528^{\circ} \mathrm{C}$.

(2) The liquidus line may be represented well by a straight line combining two points, i.e., the melting point of purified iron $\left(1538^{\circ} \mathrm{C}\right)$ and the monotectic point $\left(1528^{\circ} \mathrm{C}, 0.16^{\%}\right.$ O ).

(3) The solubility of oxygen in $\delta$-iron is $0.012 \%$ at $1528^{\circ} \mathrm{C}$.

(4) The partition ratio of oxygen $L_{0}$ is 0.076 in the temperature range between $1528^{\circ}$ and $1538^{\circ} \mathrm{C}$, independent of the concentration of oxygen in liquid iron.

\section{REFERENCES}

1) A. U. Seybolt: J. Metals, 200 (1954), 641; Trans. Met. Soc. AIME, 215 (1959), 298.

2) R. Sifferlen: Compt. Rend., 240 (1955), 2526; 244 (1957), 1192.

3) J. A. Kitchener, J. O'M. Bockris, M. Gleiser, and J. W. Evans: Acta Met., 1 (1953), 93.

4) M. Ihida, T. Ishii, S. Tsuchida, and K. Gunji: Tetsu-toHagané, 53 (1967), 394.

5) J. H.Swisher and E. T. Turkdogan: Trans. Met. Soc. AIME, 239 (1967), 426.

6) S. Tankins and N. A. Gokcen: Trans. Amer. Soc. Metals, 53 (1961), 843.

7) M. T. Hepworth, R. P. Smith, and E. T. Turkdogan: Trans. Met. Soc. AIME, 236 (1966), 1278.

8) O. Kubaschewski and E. Evans: Metallurgical Thermochemistry, (1956), 42, Pergamon Press, London.

9) J.F. Elliott, M. Gleiser, and V. Ramakrishna: Thermochemistry for Steelmaking, vol. 2 (1963), 788, AddisonWesley Publishing. 